\title{
Study on Immersive Installation that Use Interactive Media Technology
}

\author{
EunJoo Shin ${ }^{1}$ and Hyeyoung Yoo $^{2 *}$ \\ ${ }^{1}$ Interactive Design, College of Fine Art and Design, Incheon Catholic University \\ ${ }^{2 *}$ Dept. of advanced image Graduate School of Advanced Imaging Science, \\ Multimedia and Film, Chung-Ang University \\ 'ejoo@iccu.ac.kr, ${ }^{2}$ hyy345@gmail.com
}

\begin{abstract}
This paper explores the development of the Ecliptic Wall installation, which involves kinesthetic interactions and immersive experiences. Ecliptic Wall, a media art installation that uses interactive media technology, has been developed as both an artistic work and as a designed lighting unit to aid in the research of the natural phenomenon of an eclipse. The interaction technique involves the use of ultrasonic sensors, optical detectors, an LED light, a stepper motor, and a mechanical hardware for generating patterns through an LED light. This work investigates the effects of a moving light, an ambient atmosphere, and the meditative quality of immersive spaces.
\end{abstract}

Keywords: Interactive installation, kinesthetic interaction, immersive experience, artificial nature, physical computing technology

\section{Introduction}

In new media art, light is often used as a popular medium to generate visual impressions on physical structures and real spaces through the use of digital technologies and techniques. The illusion of distorting and expanding physical spaces through the use of dynamic and optical light is brought about by spatial installations based on time and ephemeral spectacles. Light can generate the illusion of a new space; further, a characteristic of light is that it can evoke a tactile illusion, i.e., the sensation of touch through visual stimuli. Thus, through the use of digital technologies, light can be used to generate immersive experiences that integrate space and time and it can also be used to generate an illusion of extending physical spaces from internal to external environment. With the advancements in digital technology in recent years, numerous media artists have explored the use of light, space, and time in large-scale architectural installations.

This research investigates the possibility of creating immersive experiences that simulate natural phenomena. This paper is a case study on the Ecliptic Wall installation, which produces an immersive experience of an artificial eclipse. In the newly created space through Ecliptic Wall installation, there are communications and interactive experiences among participants, light and space to reflect the natural phenomena.

The remainder of this paper is organized as follows. Section 2 reviews related work. Section 3 introduces the concept of the Ecliptic Wall installation, its technical operation, and the constraints of the space for the installation. Section 4 describes qualitative interactions and impressions. Finally, Section 5 concludes the paper. 


\section{Related Work}

\subsection{Falling Light}

A London based art and design studio, Troika, created Falling Light in 2010 in collaboration with Swarovski. Falling Light is an immersive installation that creates a virtual rain of light drops. It consists of 50 suspended mechanical devices each consisting of a custom-cut crystal lens, a computer programmed motor, and an LED. The white light of an LED moving closer to and further away from a lens appears as a droplet encircled by a rainbow-colored halo. The concept of evanescent and fluidity characteristic of raindrops is achieved by generating, for viewers, an experience of immersive showers through lights. Even though Falling Light looks like a kinesthetic installation, it is a highly technology driven installation that uses physical computing technology to control custom-made hardware, and thereby, it creates a new experience through light effects.
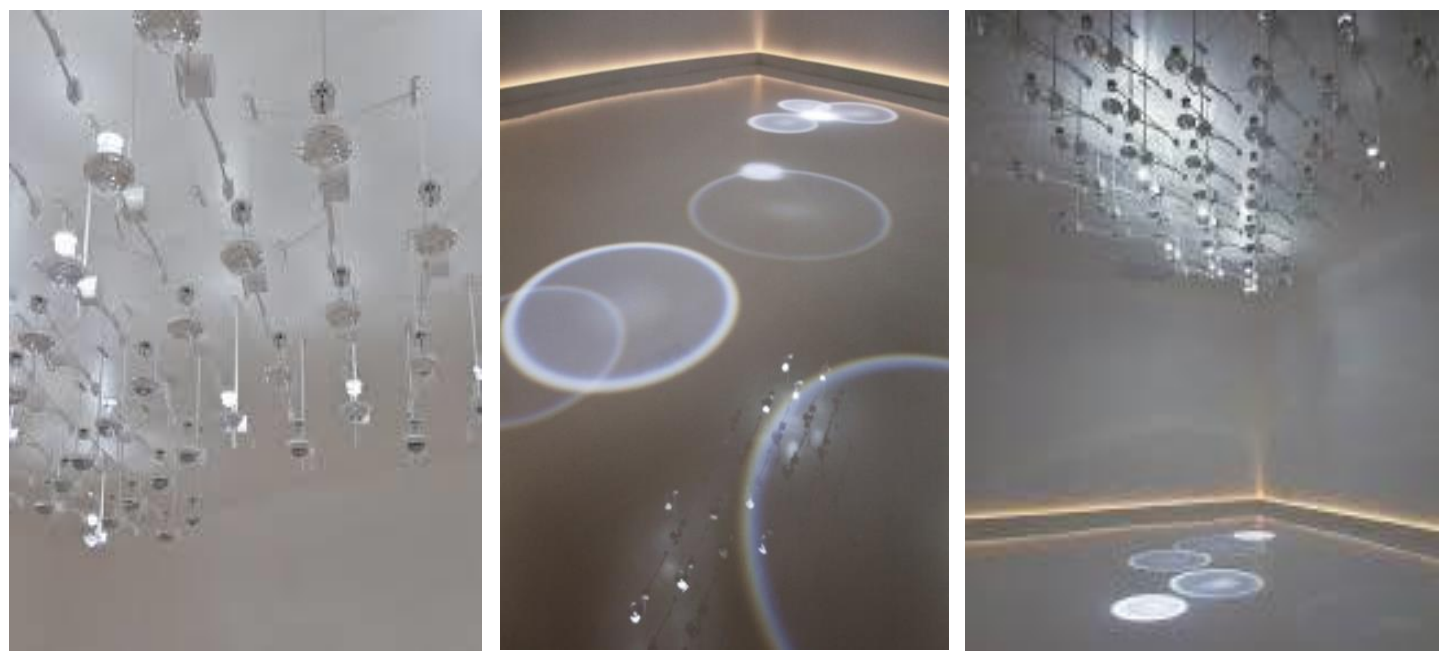

Figure 1. Falling Light, 2010

\subsection{Solar Equation}

Solar Equation, the work of media artist Rafael Lozano-Hemmer, is a technologically advanced interactive installation in a public space. It appears as a giant sun looming above the Winter Festival in Melbourne. The artwork features a large glowing spherical balloon that is $14 \mathrm{~m}$ in diameter, which is custom-manufactured for five HD projectors that produce light effects on it. All the activities that occur on the surface of the sun like turbulence, flares, and sunspots are reproduced through animations on the balloon. The solar animations involve constantly changing images that never repeat. Viewers can experience solar phenomena that occur on the surface of the sun as well as control the animations on the balloon in real-time by selecting different dynamic visualizations through applications on an iPhone, iPod touch, or iPad. Thus, Solar Equation reproduces solar phenomena for viewers in a public site and also allows them to view their preferred solar phenomenon by selecting the same through hands-on smart devices. This is made possible by digital technologies. 


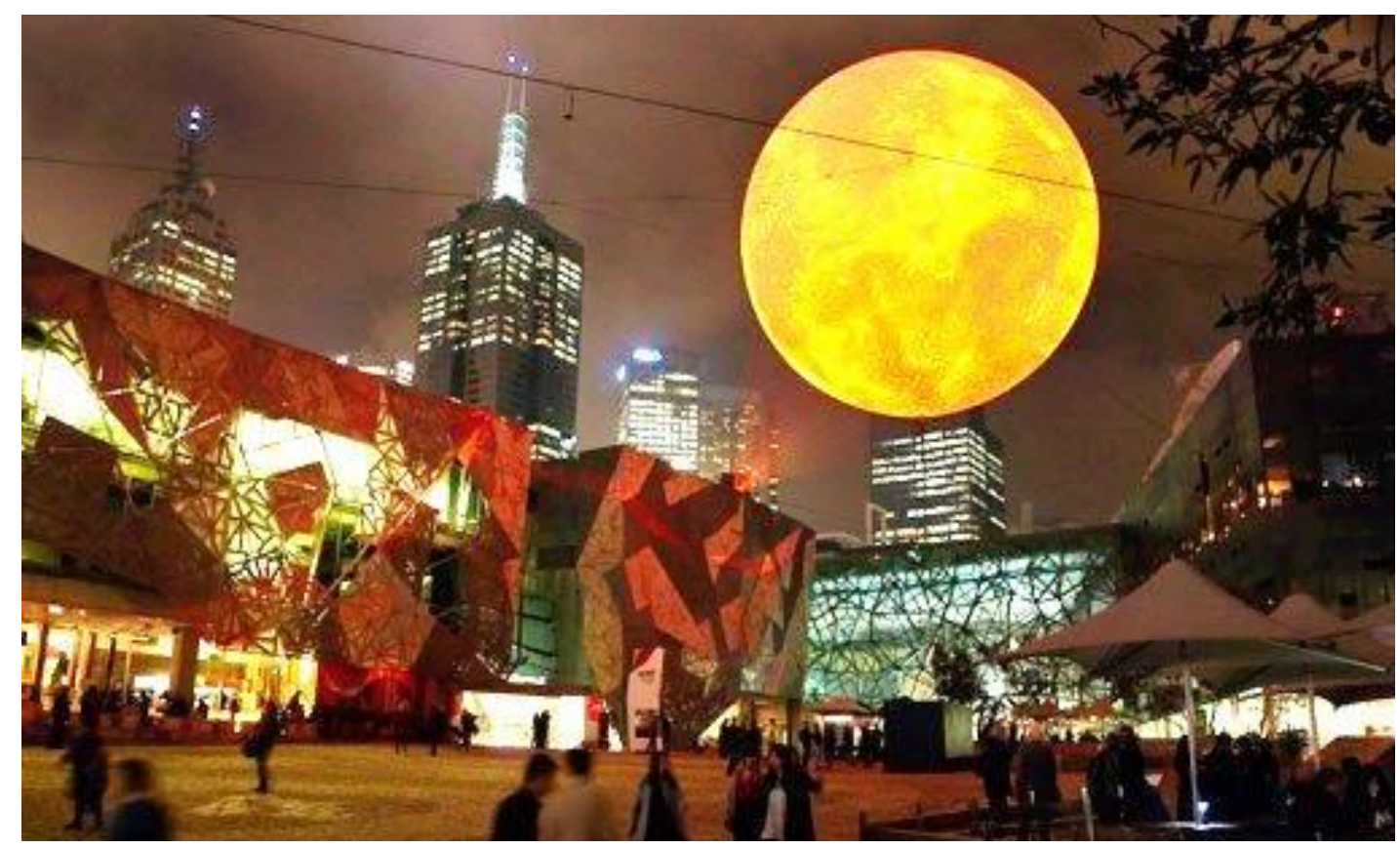

Figure 2. Solar Equation, 2011

\subsection{Solar Equation}

Living Light is a permanent outdoor pavilion by David Benjamin and Soo-in Yang that is located in Peace Park, across the World Cup Stadium in Seoul, Korea. It consists of 27 blocks that glow and blink in response to both air monitoring information and public interest in an area. Together, the blocks look like a tree shaped glass canopy and each block represents one of the 27 neighborhood boundaries of Seoul city. People can receive air quality data about a specific section of Seoul by sending a text message to the structure. The corresponding block will then glow in response to the people interaction and text back the feedback data of the air quality to those who are interested in receiving air quality statistics in real time. People can also enjoy the visual appeal of illuminating light patterns of a map of Seoul by viewing it from within the pavilion or simply from nearby streets and buildings. Thus, Living Light, as an outdoor pavilion, represents a novel way of communicating with the public in a public space. 


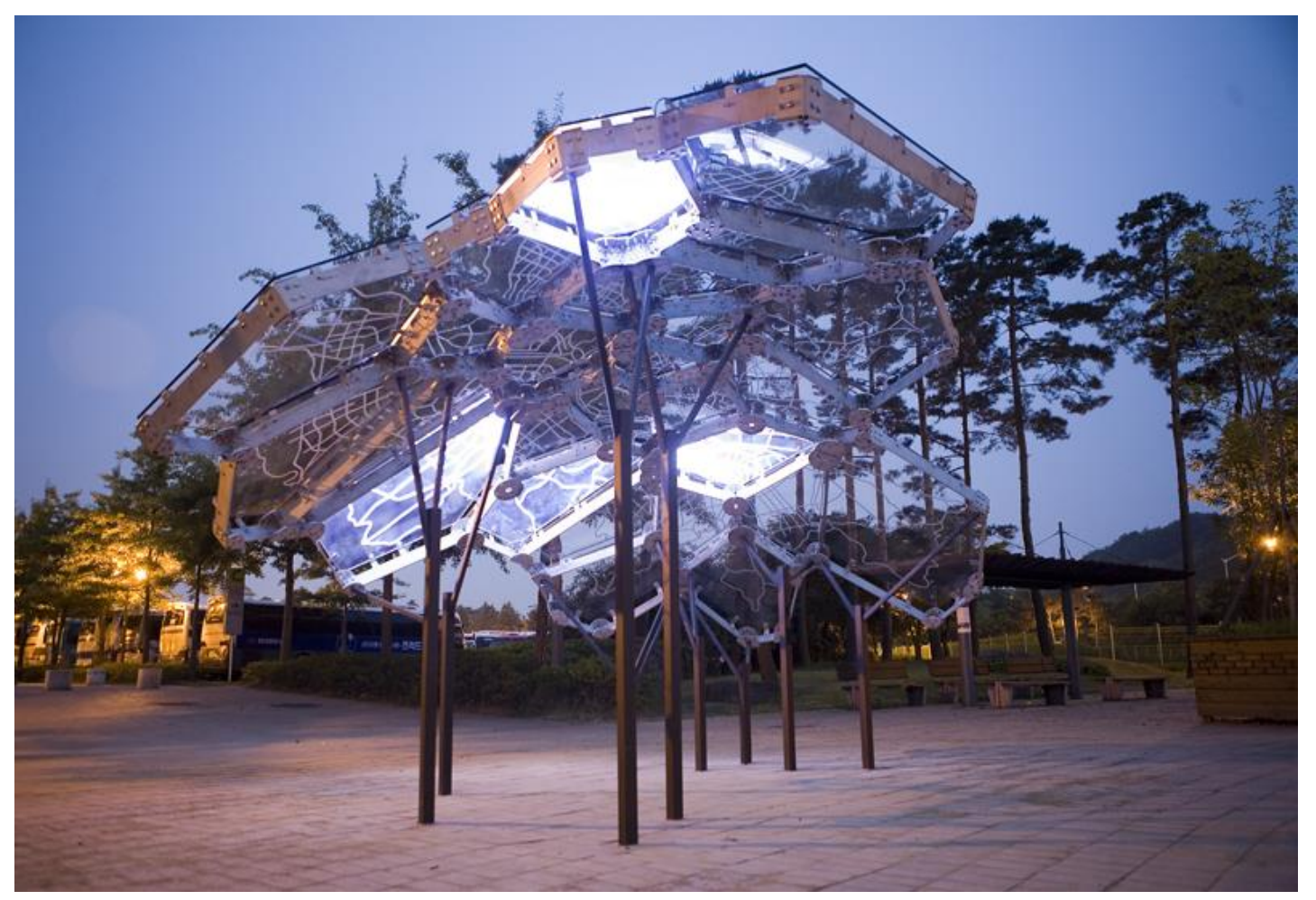

Figure 3. Living Light, 2011

\section{Interactive Installation and Technical Detail}

\subsection{Background}

The increasing affordability of physical computing technologies such as sensors and microcontrollers has created new opportunities for creating interactive environments. Such opportunities for interaction are realized through electronic, artistic installations. Even though artistic installations serve as an interface between the media and audience, they are designed to facilitate interactions in response to changes in their surrounding as well as handle variations in audience flow and audience engagement in real-time. In particular, lighting has often been used to create an optical spectacle for immersive experiences and media artists have explored its use in large-scale installations through the use of physical computing technologies and techniques. Although numerous installations that produce an optical spectacle exist, only few of those focus on producing immersive experiences based on natural phenomena.

Ecliptic Wall artificially reproduces the characteristics of the natural phenomenon of an eclipse through dynamic lighting produced by an interactive installation that consists of electronic motors and sensors controlled. Furthermore, it explores the synergy between an immersive experience based on a natural phenomenon, an ambient atmosphere, and aesthetic and kinesthetic interaction.

\subsection{Configuration}

The installation interprets an eclipse as perceived by the related ephemeral movement of light. Ecliptic Wall produces the visual effect of a lunar eclipse by applying the positional 
changes of the moon in the case of an eclipse to the design of the installation's embodied interface, which includes a lighting device and a mechanical structure.

To correctly observe the effect, audiences are asked to stand facing the structure in a straight line. Next, lights in the space around them are turned off. An important aesthetic consideration in designing the space and interactive experience is that when an eclipse occurs the sun, earth, and moon are relatively aligned; artificially reproducing the conditions of the same is essential. To realize the latter, a lighting device and a customized hardware are developed. Further, the explorative nature of this interaction invites audiences to learn how the space reacts.

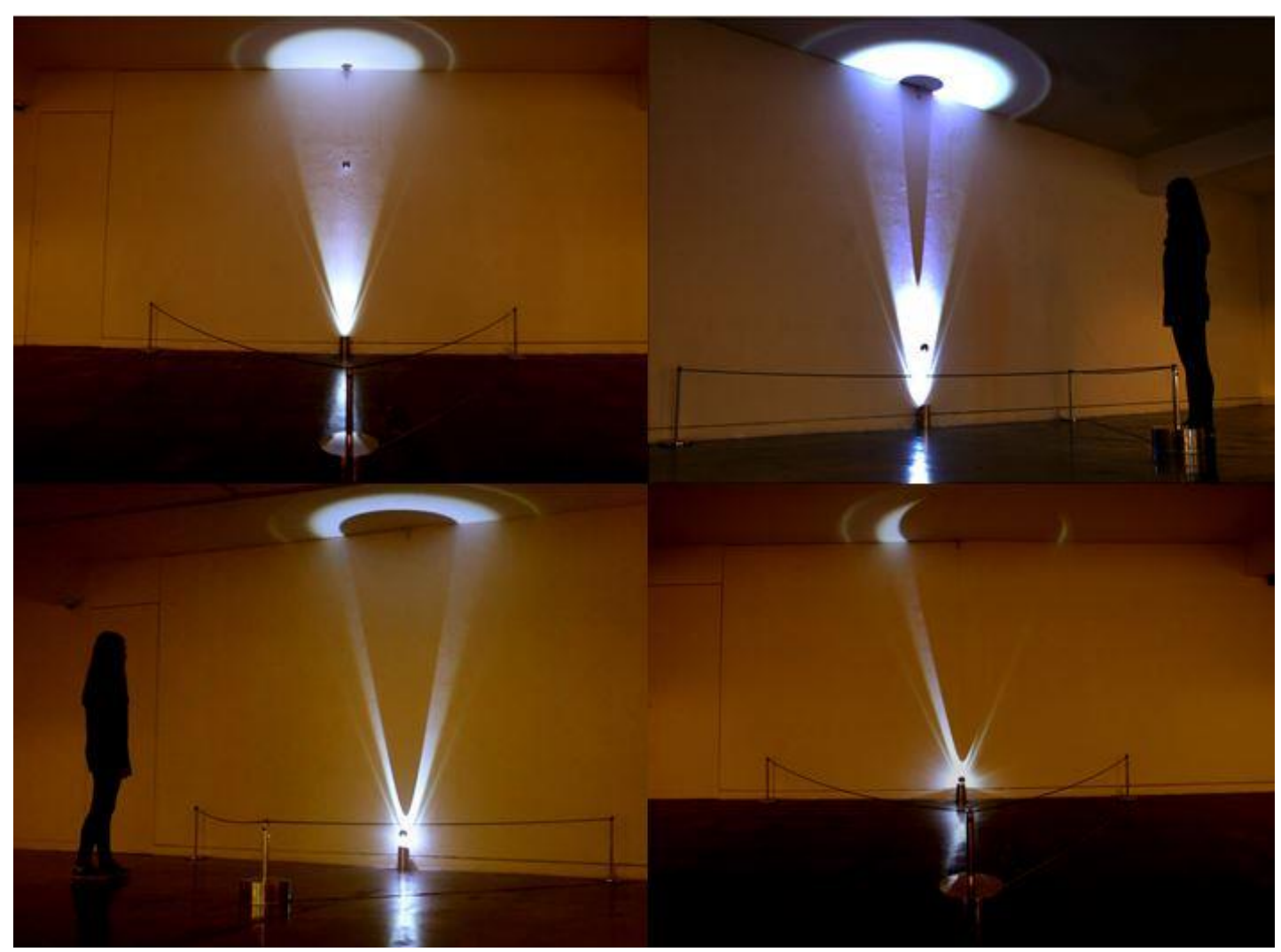

Figure 4. Ecliptic Wall, 2013

\subsection{Technical Operation}

Custom-made metal rod contains an ultrasonic sensor, an Arduino processor, a stepper motor, and a mechanical hardware that holds a wire connected to a steel ball through a small wall-mounted roller. Another cup-shaped steel structure on the floor holds an LED light and a polished inner surface to reflect the lighting. The data from the ultrasonic sensor is digitized by an Arduino processor and is used to control the speed of the stepper motor. The signal from two optical detectors inside a cup-shaped steel structure and a small wall-mounted roller each trigger the motor to either run or stop. Thus, computer programmed hardware controls the motion of a steel ball that generates blue lighting textures and shadows. 

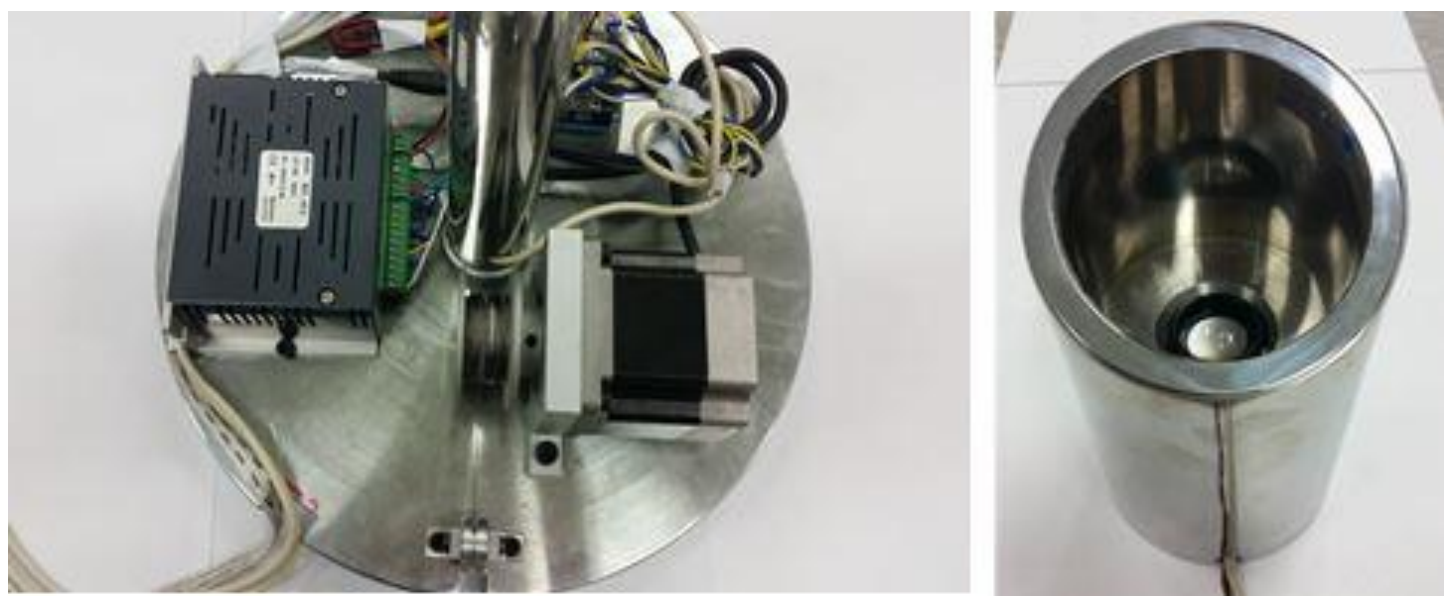

Figure 5. Customized Metal Rod and Cup-Shaped Metal Structure

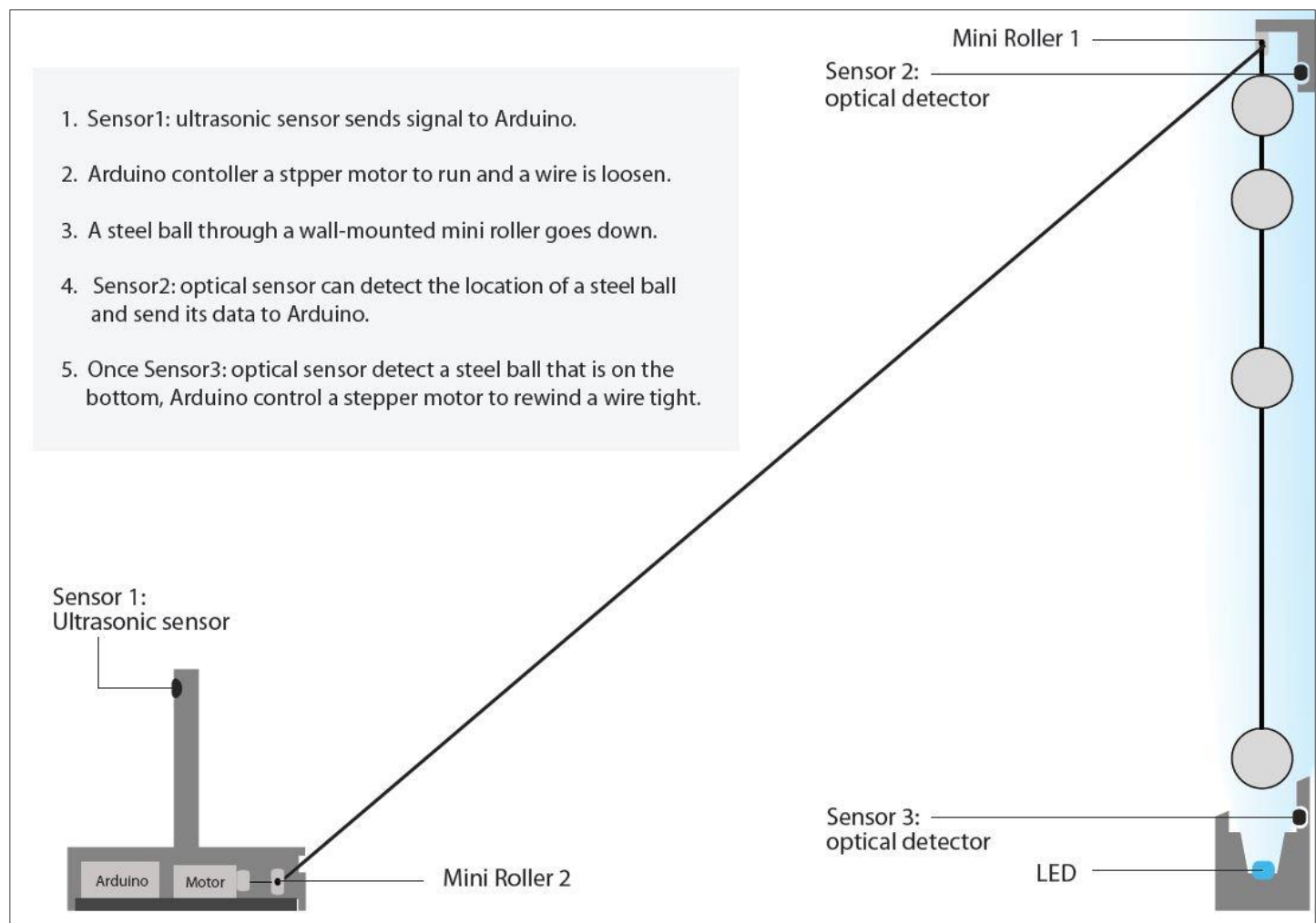

Figure 6. Technical Diagram

\subsection{Space}

The Ecliptic Wall installation requires a minimum space of $4 \mathrm{~m} * 3 \mathrm{~m} * 2.3 \mathrm{~m}(\mathrm{~h})$. The moving ball of the installation needs to be suspended in the middle and the main controller device needs to be installed at the bottom in the center of the installation. In addition, the wall point should be strong enough to hold the weight of a metal ball. The installation produces quiet ambient clinking sounds, and therefore, must be installed away from loud-sound installations. 


\section{Interaction and Impressions}

A moving steel ball suspended from the top of a wall produces shadows and the corresponding light effect results in an immersive experience for the audience. The movement of the steel ball represents the conceptual movement of the moon, which results in the immersive experience of an eclipse. The installation consists of a steel ball moving up and down along a wall, a computer programmed stepper motor, an LED illuminating from the bottom, optical detectors, and ultrasonic sensors inside three metal rods on three vertexes of a triangular lined area. When audiences move closer to the triangular area, the ball moves down toward a light on the floor, obstructing the light and producing darkness all around. Subsequently, it moves slowly up and produces bluish light reflections.

Ecliptic Wall was installed at the exhibition RESONATE: Media Spaces and Installations, at Gallery Palais de Seoul. During the exhibition, subtle effects are produced through implicit mechanisms and the space around Ecliptic Wall is dynamically transformed leaving a deceptively simple experience of the sublime. We also found that Ecliptic Wall reduces direct audience interaction in favor of a more ambient mode of interaction.
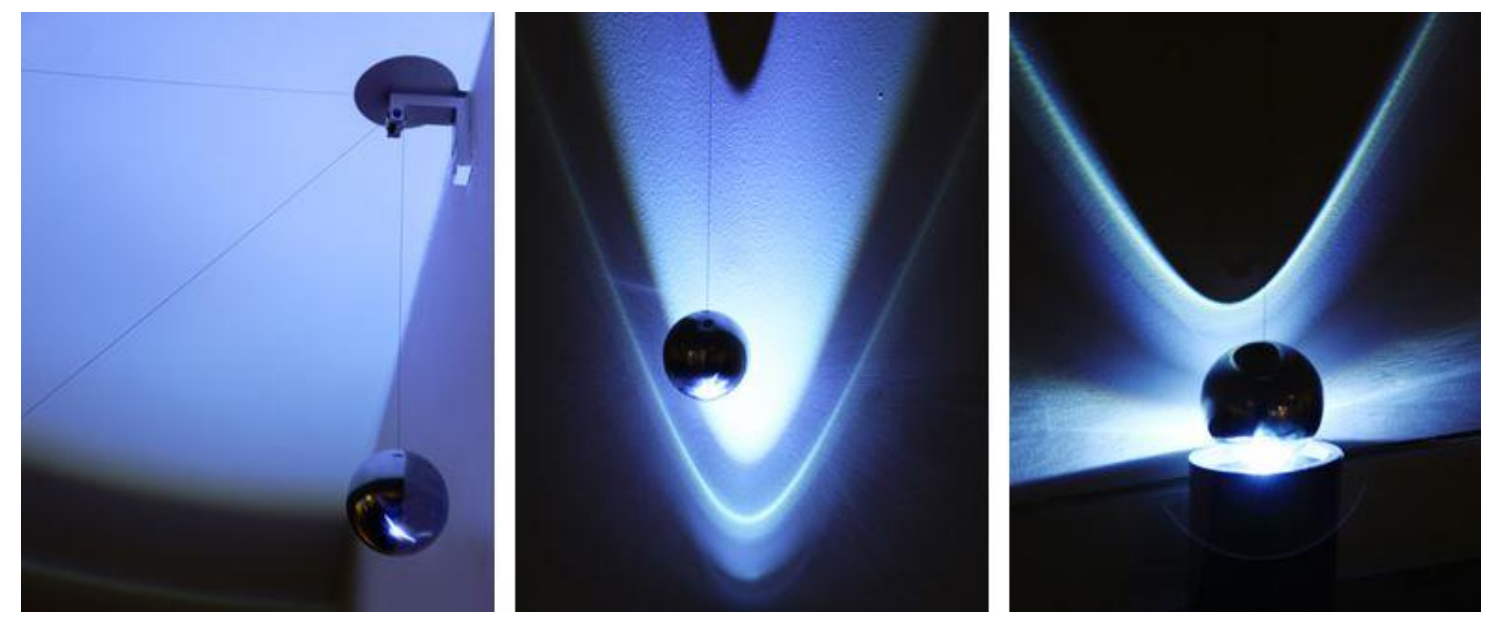

Figure 7. Customized Hardware

\section{Conclusions and Future Work}

In this case study, we reviewed Ecliptic Wall, which has been developed as both an artistic installation and as a designed lighting device to aid in the research of the natural phenomenon, of an eclipse, through the progress of computational technologies. Its design has been inspired from art methodologies, technical experience, and interaction design. In this study, we examined the use of implicit and embodied interface for controlling lighting and immersive experiences so as to determine new ways of interacting with light. In the future, we plan to conduct studies on large-scale installations in public spaces and interactive media designs that involve collective participation to let them engage with a follow-up project in a more active and direct manner. We believe the findings of this case study can significantly assist in future designs of customized participatory experiences in public spaces in terms of clear strategies for highlighting the interface of the extent different digital technology and spatial configuration. 


\section{References}

[1] N. Oliveira and N. Oxley, "in Installation Art", Thames and Hudson, London, (1996), pp. 14-76

[2] B. Jan, "The Art of Light and Space", Abbeville, New York, (1996), pp. 8-45.

[3] P. Christiane, "Digital Art", Thames and Hudson, London, (2008), pp. 71-78.

[4] K. J. Kortbek and K. Grønbæk, "Interactive spatial multimedia for communication of art in the physical museum space", ACM, vol. 08, (2008), pp. 609-618.

[5] A. Rowe, "Within an Ocean of light: creating volumetric light scape", Proc. SIGGRAPH '12, (2012), pp. 358365.

[6] G. Monaci, T. Gritti, F. Vignoli, W. Walmink and M. Hendriks, "Flower power", Proc. $19^{\text {th }}$ ACM international conference on Multimedia, (2011), pp. 909-912.

[7] E. Shin and H. Yoo, "Ecliptic Wall: Interactive Installation for Immersive Experience", Proc. ASTL,SERSC, vol. 67, (2014), pp. 1-4.

[8] "Troika's website", http://www.troika.uk.com/work/falling-light/.

[9] "Rafael Lozano Hemmer's website", www.lozano-hemmer.com/flatsun.php.

[10] "Living Light's website”, http://www.livinglightseoul.net/.

[11] “Arduino's website”, http://arduino.cc.

\section{Authors}

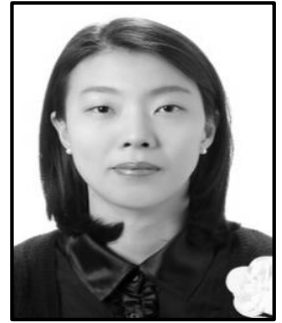

EunJoo Shin, she studied Art and Computational Technologies at Goldsmith, University of London as a Ph.D. candidate (finished 2014). Currently, she works an assistant professor of Interaction Design at College of Fine Art and Design, Incheon Catholic University since 2012. Her research interests are in the areas of human computer interaction and embodied hardware through creative and social use of technology.

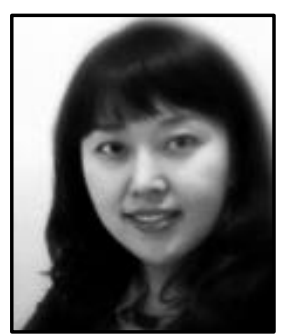

Hyeyoung Yoo, she received the $\mathrm{Ph} D$ degree in Art \& Technology at Graduate School of Advanced Imaging Science, Multimedia \& Film in Chung-Ang University, Seoul, Korea, in 2015. Currently, she is an adjunct professor at Hanyang Woman's University. Her research interests include volumetric display, hologram and data visualization. She won the grand prize of the 2014 HCI Korea creative award. 